\title{
TEMPERATURE, URBANIZATION AND BODY COLOR POLYMORPHISM IN SOUTH BRAZILIAN POPULATIONS OF DROSOPHILA KIKKAWAI (DIPTERA, DROSOPHILIDAE)
}

\author{
Bartira E. Pinheiro da Costa ${ }^{1}$ \\ Cláudia Rohde ${ }^{2}$ \\ Vera Lúcia da Silva Valente ${ }^{2}$
}

\begin{abstract}
Body color polymorphism of urban populations of cosmopolite fly Drosophila kikkawai Burla, 1954 was investigated in relation to its possible association with environmental temperature. Samples of D. kikkawai were collected in spring, summer, autumn and winter between 1987 to 1988 , in zones with different levels of urbanization in the southern Brazilian city of Porto Alegre, Rio Grande do Sul. A clear association was observed between darker flies and both seasons with low temperatures and areas of low urbanization (where temperature is generally lower than in urbanized areas). Results of preliminary laboratory experiments involving six generations of flies grown in chambers at temperatures of $17^{\circ}$ and $25^{\circ} \mathrm{C}$ confirmed this tendency to a relationship between body color and temperature, with allele frequency of the main gene involved in body pigmentation changing over time.
\end{abstract}

KEYWORDS. Drosophila, urban populations, color polymorphism, temperature.

\section{INTRODUCTION}

Drosophila kikkawai Burla, 1954 is a cosmopolitan species of fruit fly (PINHEIRO \& VALENTE, 1993). It belongs to the D. melanogaster species group and occurs both in domestic and disturbed non-domestic sites. TsaCAS \& DAVID (1978) suggested that the introduction of this species to South America and Africa occurred prior to, and independent of human activity, and AsHBURNER (1989) considered that this species had only recently spread from its origin in Southeast Asia to South America and Africa. This species is of interest to evolutionists because it presents discrete genetic polymorphisms, including various types of karyotypes (BAIMAI et al.,1986) and variable posterior abdominal segment pigmentation (Freire-MaiA, 1953). Freire-MAiA \& Freire-MAiA (1964)

\footnotetext{
1. Instituto de Pesquisas Biomédicas, Pontifícia Universidade Católica do Rio Grande do Sul, Av. Ipiranga, 6690, 90610-000, Porto Alegre, RS, Brazil.

2. Departamento de Genética, Instituto de Biociências, Universidade Federal do Rio Grande do Sul, Caixa Postal 15053, 91501-970, Porto Alegre, RS, Brazil. (vera.gaiesky@ufrgs.br)
} 
observed higher frequencies of light-colored D. kikkawai females in populations collected in hot climates at different latitudes. It is also known that in some other Drosophila species, darker forms occur more commonly in samples collected in colder climates (HEED \& BlaKe, 1963; LeE, 1963; GiBERT et al., 1996, 1998; MACHADO et al., 2001). There are two different systems for explaining the abdominal color polymorphism in D. kikkawai, the earlier being that of Freire-MAIA et al. (1954) and Freire-MAIA (1964a), which states that geographical genetic differences in modifier genes affect this character and produce three phenotypes, dark/dark (CC genotype), dark/light (Cc genotype) and light/light (cc genotype). A more recent system is that of GIBERT et al. (1999), who worked with Indian populations of D. kikkawai and suggested co-dominance of the major gene involved in abdominal segment color and modifier gene effects to explain the dark/dark (DD), dark/ light (DL) and light/light (LL), phenotypes found in their studies.

In the southern Brazilian city of Porto Alegre, D. kikkawai is common in Drosophila assemblies where it is sympatric with some wild species that are in the process of colonizing the urban environment (VALENTE et al., 1989). Porto Alegre is located in the coldest area of Brazil, has four well-defined seasons and has a known urbanization pattern. The subject was an analysis of the variations of abdominal segment pigmentation and fluctuations of D. kikkawai populations in relation to environmental variables in Porto Alegre and attempts to detect some type of association between these variables as a function of time and the level of disturbance (urbanization) of the collection sites.

\section{MATERIAL AND METHODS}

Fruit fly populations were sampled by the authors in Porto Alegre city $\left(30^{\circ} 02^{\prime} \mathrm{S} ; 51^{\circ} 31^{\prime} \mathrm{W}\right.$; roughly 1,3 million inhabitants) between January 1987 and December 1988 by collecting pre-adults (eggs, larvae and pupae) from rotten fruits and trapping adult flies on closed banana baits (model of Tidon \& SENE, 1988) placed in parks, squares and streets along transects correspondent to the main avenues of the city, in three different urbanization zones characterized by RUszczYK (1986/1987) in the 1980 decade: a highly urbanized zone mainly occupied by buildings where cover vegetation is less than $20 \%$ (high urbanization, $\mathrm{HU}$ ); a zone of medium urbanization containing houses and some buildings and having 20 to $40 \%$ of cover vegetation (medium urbanization, MU); and a zone of low urbanization predominantly occupied by houses and having more than $40 \%$ cover vegetation (low urbanization, LU) (fig. 1). Urbanization characteristics of the sampling site (percentage vegetation cover, distance from the center of the city, altitude) were recorded (tab. I). Climatic data (temperature, relative humidity and rainfall scores during the month of collection and the preceding month) for Porto Alegre were obtained from the $8^{\circ}$ Distrito de Meteorologia, Ministério da Agricultura do Brasil.

Drosophila kikkawai samples were sorted by sex and phenotypic color (dark, medium and light, fig. 2-7), based on the color of their abdominal segments, according to the system of FreIREMaia et al. (1954) and Freire-Maia (1964a). Specimens testimony of these samples are deposited in the Drosophilid collection of the Departamento de Genética, Universidade Federal do Rio Grande do Sul (UFRGS).

Laboratory experiments were also performed to investigate the effect of temperature on abdominal color polymorphism in these populations. Seven virgin females of each phenotype were mated with seven males of the three different phenotypes to form three populations. This was done by mating $\mathrm{CC}, \mathrm{Cc}$ and $\mathrm{cc}$ females with $\mathrm{CC}$ males (population 1, P1), Cc males (population 2, P2) and cc males (population 3, P3). Three replicates of all crosses and populations were maintained in chambers at constant temperatures of $25^{\circ}$ or $17^{\circ} \mathrm{C} \pm 1^{\circ} \mathrm{C}$ and $60 \%$ relative humidity, respectively, on standard culture medium (Marques et al., 1966). To obtain the next generations (G1-G6) the adult flies were transferred to new vials with fresh food, three times. The adult progeny was allowed to interbreed to produce the next generation and then their abdominal color polymorphism were analyzed. 
Table I. Original data used to perform the multiple regression analysis of Drosophila kikkawai frequency and weather and urban action variables. Samples 1 to 44 were collected during 1982 to January 1987; samples 45 to 79 were collected during February 1987 to 1988 (S, sample; F, frequency (\%) of flies; TEMP ANT MAX, TEMP ANT MIN, previous temperature of collection, maximum average, minimum average; TEMP COL MAX, TEMP COL MIN, temperature of the month of collection, maximum average, minimum average; RH ANT, previous humidity of collection; RH COL, relative humidity of the month of collection; RH ANT, previous rainfall of collection; RH COL, rainfall of the month of collection; \% VEG, percentual vegetation; DIST, distance from downtown to collection point; ALT, altitude).

\begin{tabular}{|c|c|c|c|c|c|c|c|c|c|c|c|c|}
\hline $\mathrm{S}$ & $\mathrm{F}$ & $\begin{array}{c}\text { TEMP } \\
\text { ANT } \\
\text { MAX }\end{array}$ & $\begin{array}{l}\text { TEMP } \\
\text { ANT } \\
\text { MIN }\end{array}$ & $\begin{array}{c}\text { TEMP } \\
\text { COL } \\
\text { MAX }\end{array}$ & $\begin{array}{l}\text { TEMP } \\
\text { COL } \\
\text { MIN }\end{array}$ & $\begin{array}{c}\text { RH } \\
\text { ANT }\end{array}$ & $\begin{array}{c}\mathrm{RH} \\
\mathrm{COL}\end{array}$ & $\begin{array}{c}\text { RF } \\
\text { ANT }\end{array}$ & $\begin{array}{c}\mathrm{RF} \\
\mathrm{COL}\end{array}$ & $\begin{array}{c}\% \\
\text { VEG }\end{array}$ & DIST & ALT \\
\hline 01 & 16.43 & 21.6 & 12.9 & 23.3 & 14.5 & 78 & 78 & 195.4 & 193.2 & 20 & 3.3 & 41 \\
\hline 02 & 19.28 & 25.3 & 16.9 & 21.9 & 11.6 & 78 & 79 & 130.7 & 110.9 & 20 & 3.4 & 33 \\
\hline 03 & 18.63 & 21.9 & 11.6 & 20.1 & 11.6 & 79 & 83 & 110.9 & 109.9 & 20 & 3.4 & 33 \\
\hline 04 & 35.79 & 20.1 & 11.6 & 21.1 & 11.0 & 83 & 79 & 109.9 & 108.8 & 20 & 3.3 & 41 \\
\hline 05 & 25.40 & 20.1 & 11.6 & 21.1 & 11.0 & 83 & 79 & 109.9 & 108.8 & 20 & 2.0 & 19 \\
\hline 06 & 7.49 & 20.1 & 11.6 & 21.1 & 11.0 & 83 & 79 & 109.9 & 108.8 & 30 & 7.0 & 11 \\
\hline 07 & 18.63 & 22.2 & 14.5 & 25.7 & 15.9 & 78 & 75 & 150.6 & 63.9 & 30 & 7.0 & 11 \\
\hline 08 & 5.07 & 25.7 & 15.9 & 29.5 & 18.1 & 75 & 66 & 63.9 & 229.0 & 50 & 8.0 & 3 \\
\hline 09 & 22.22 & 29.5 & 18.1 & 29.5 & 19.3 & 66 & 70 & 229.0 & 161.6 & 20 & 3.4 & 33 \\
\hline 10 & 11.39 & 29.5 & 18.1 & 29.5 & 19.0 & 66 & 70 & 229.0 & 161.6 & 50 & 8.0 & 3 \\
\hline 11 & 4.25 & 29.5 & 19.3 & 31.7 & 21.3 & 70 & 69 & 161.6 & 39.3 & 50 & 5.4 & 35 \\
\hline 12 & 21.30 & 29.5 & 19.3 & 31.7 & 21.3 & 70 & 69 & 161.6 & 39.3 & 10 & 0.2 & 5 \\
\hline 13 & 17.05 & 29.5 & 19.3 & 21.7 & 21.3 & 70 & 69 & 161.6 & 39.3 & 20 & 1.1 & 10 \\
\hline 14 & 35.24 & 29.5 & 19.3 & 31.7 & 21.3 & 70 & 69 & 161.6 & 39.3 & 50 & 7.0 & 50 \\
\hline 15 & 5.74 & 29.5 & 19.3 & 31.7 & 21.3 & 70 & 69 & 161.6 & 39.3 & 10 & 2.3 & 12 \\
\hline 16 & 28.18 & 29.5 & 19.3 & 31.7 & 21.3 & 70 & 69 & 161.6 & 39.3 & 10 & 0.3 & 5 \\
\hline 17 & 55.67 & 29.5 & 19.3 & 31.7 & 21.3 & 70 & 69 & 161.6 & 39.3 & 10 & 2.5 & 15 \\
\hline 18 & 16.74 & 29.5 & 19.3 & 31.7 & 21.3 & 70 & 69 & 161.6 & 39.3 & 50 & 4.2 & 35 \\
\hline 19 & 7.92 & 29.5 & 19.3 & 31.7 & 21.3 & 70 & 69 & 161.6 & 39.3 & 30 & 6.5 & 11 \\
\hline 20 & 30.92 & 31.7 & 21.3 & 29.8 & 20.6 & 69 & 73 & 39.3 & 141.3 & 45 & 7.7 & 2 \\
\hline 21 & 6.02 & 29.8 & 20.6 & 27.6 & 18.6 & 73 & 75 & 141.3 & 137.1 & 20 & 3.3 & 41 \\
\hline 22 & 17.15 & 29.8 & 20.6 & 27.6 & 18.6 & 73 & 75 & 141.3 & 137.1 & 20 & 3.4 & 33 \\
\hline 23 & 20.79 & 29.8 & 20.6 & 27.6 & 18.6 & 73 & 75 & 141.3 & 137.1 & 45 & 7.7 & 2 \\
\hline 24 & 9.7 & 29.8 & 20.6 & 27.6 & 18.6 & 73 & 75 & 141.3 & 137.1 & 30 & 7.0 & 11 \\
\hline 25 & 2.50 & 27.6 & 18.6 & 25.9 & 18.1 & 75 & 80 & 137.1 & 156.8 & 20 & 5.8 & 11 \\
\hline 26 & 7.71 & 27.6 & 18.6 & 25.9 & 18.1 & 75 & 80 & 137.1 & 156.8 & 20 & 3.4 & 33 \\
\hline 27 & 6.55 & 27.6 & 18.6 & 25.9 & 18.1 & 75 & 80 & 137.1 & 156.8 & 20 & 3.5 & 38 \\
\hline 28 & 26.64 & 22.0 & 12.9 & 22.3 & 12.3 & 78 & 82 & 164.4 & 61.3 & 20 & 5.8 & 11 \\
\hline 29 & 6.55 & 22.0 & 12.9 & 22.3 & 12.3 & 78 & 82 & 164.4 & 61.3 & 50 & 12.2 & 8 \\
\hline 30 & 30.85 & 22.3 & 12.3 & 20.3 & 10.4 & 82 & 79 & 61.3 & 126.5 & 20 & 5.8 & 11 \\
\hline 31 & 15.34 & 22.3 & 12.3 & 20.3 & 10.4 & 82 & 79 & 61.3 & 126.5 & 20 & 3.4 & 33 \\
\hline 32 & 3.53 & 22.3 & 12.3 & 20.3 & 10.4 & 82 & 79 & 61.3 & 126.5 & 30 & 4.3 & 75 \\
\hline 33 & 9.10 & 22.3 & 12.3 & 20.3 & 10.4 & 82 & 79 & 61.3 & 126.5 & 50 & 5.4 & 35 \\
\hline 34 & 11.83 & 22.3 & 12.3 & 20.3 & 10.4 & 82 & 79 & 61.3 & 126.5 & 10 & 5.4 & 35 \\
\hline 35 & 14.42 & 22.3 & 12.3 & 20.3 & 10.4 & 82 & 79 & 61.3 & 126.5 & 10 & 0.3 & 5 \\
\hline 36 & 8.13 & 22.3 & 12.3 & 20.3 & 10.4 & 82 & 79 & 61.3 & 126.5 & 10 & 3.6 & 3 \\
\hline 37 & 18.43 & 22.3 & 12.3 & 20.3 & 10.4 & 82 & 79 & 61.3 & 126.5 & 50 & 38.0 & 2 \\
\hline 38 & 8.13 & 22.3 & 12.3 & 20.3 & 10.4 & 82 & 79 & 61.3 & 126.5 & 30 & 7.0 & 11 \\
\hline 39 & 3.80 & 24.0 & 14.3 & 26.5 & 17.6 & 71 & 74 & 138.1 & 283.4 & 30 & 4.6 & 75 \\
\hline 40 & 9.63 & 28.5 & 19.2 & 29.4 & 20.0 & 70 & 74 & 103.3 & 170.0 & 50 & 12.2 & 8 \\
\hline
\end{tabular}


Table I. (continued)

\begin{tabular}{|c|c|c|c|c|c|c|c|c|c|c|c|c|}
\hline $\mathrm{S}$ & $\mathrm{F}$ & $\begin{array}{l}\text { TEMP } \\
\text { ANT } \\
\text { MAX }\end{array}$ & $\begin{array}{l}\text { TEMP } \\
\text { ANT } \\
\text { MIN }\end{array}$ & $\begin{array}{l}\text { TEMP } \\
\text { COL } \\
\text { MAX }\end{array}$ & $\begin{array}{l}\text { TEMP } \\
\text { COL } \\
\text { MIN }\end{array}$ & $\begin{array}{c}\text { RH } \\
\text { ANT }\end{array}$ & $\begin{array}{c}\mathrm{RH} \\
\mathrm{COL}\end{array}$ & $\begin{array}{c}\text { RF } \\
\text { ANT }\end{array}$ & $\begin{array}{c}\mathrm{RF} \\
\mathrm{COL}\end{array}$ & $\begin{array}{c}\% \\
\text { VEG }\end{array}$ & DIST & ALT \\
\hline 41 & 69.30 & 28.5 & 19.2 & 29.4 & 20.0 & 70 & 74 & 103.3 & 170.0 & 20 & 1.1 & 10 \\
\hline 42 & 59.15 & 28.5 & 19.2 & 29.4 & 20.0 & 70 & 74 & 103.3 & 170.0 & 50 & 7.0 & 50 \\
\hline 43 & 35.24 & 28.5 & 19.2 & 29.4 & 20.0 & 70 & 74 & 103.3 & 170.0 & 10 & 2.3 & 12 \\
\hline 44 & 43.97 & 28.5 & 19.2 & 29.4 & 20.0 & 70 & 74 & 103.3 & 170.0 & 10 & 1.3 & 20 \\
\hline 45 & 11.97 & 30.3 & 20.7 & 29.9 & 19.0 & 75 & 75 & 118.2 & 89.0 & 10 & 3.6 & 3 \\
\hline 46 & 4.37 & 30.3 & 20.7 & 29.9 & 19.0 & 75 & 75 & 118.2 & 89.0 & 30 & 3.7 & 55 \\
\hline 47 & 12.52 & 30.3 & 20.7 & 29.9 & 19.0 & 75 & 75 & 118.2 & 89.0 & 50 & 12.2 & 8 \\
\hline 48 & 58.50 & 29.9 & 19.0 & 25.9 & 17.4 & 75 & 80 & 89.0 & 114.6 & 10 & 2.3 & 12 \\
\hline 49 & 26.06 & 29.9 & 19.0 & 25.9 & 17.4 & 75 & 80 & 89.0 & 114.6 & 10 & 1.3 & 20 \\
\hline 50 & 16.22 & 29.9 & 19.0 & 25.9 & 17.4 & 75 & 80 & 89.0 & 114.6 & 30 & 4.4 & 39 \\
\hline 51 & 13.81 & 29.9 & 19.0 & 25.9 & 17.4 & 75 & 80 & 89.0 & 114.6 & 50 & 9.6 & 18 \\
\hline 52 & 4.37 & 29.9 & 19.0 & 25.9 & 17.4 & 75 & 80 & 89.0 & 114.6 & 50 & 9.6 & 18 \\
\hline 53 & 5.74 & 29.9 & 19.0 & 25.9 & 17.4 & 75 & 80 & 89.0 & 114.6 & 30 & 3.3 & 55 \\
\hline 54 & 3.34 & 29.9 & 19.0 & 25.9 & 17.4 & 75 & 80 & 89.0 & 114.6 & 20 & 1.1 & 10 \\
\hline 55 & 3.34 & 29.9 & 19.0 & 25.9 & 17.4 & 75 & 80 & 89.0 & 114.6 & 30 & 7.8 & 0 \\
\hline 56 & 4.05 & 29.9 & 19.0 & 25.9 & 17.4 & 75 & 80 & 89.0 & 114.6 & 50 & 9.6 & 18 \\
\hline 57 & 18.72 & 25.9 & 17.4 & 19.7 & 10.7 & 80 & 78 & 114.6 & 200.9 & 20 & 2.1 & 19 \\
\hline 58 & 23.58 & 25.9 & 17.4 & 19.7 & 10.7 & 80 & 78 & 114.6 & 200.9 & 20 & 3.3 & 41 \\
\hline 59 & 15.68 & 25.9 & 17.4 & 19.7 & 10.7 & 80 & 78 & 114.6 & 200.9 & 50 & 4.2 & 35 \\
\hline 60 & 4.33 & 19.7 & 10.7 & 19.1 & 8.2 & 78 & 80 & 200.9 & 82.9 & 20 & 2.1 & 19 \\
\hline 61 & 1.90 & 19.7 & 10.7 & 19.1 & 8.2 & 78 & 80 & 200.9 & 82.9 & 20 & 5.8 & 11 \\
\hline 62 & 5.74 & 23.8 & 14.6 & 26.6 & 17.3 & 73 & 74 & 102.5 & 170.4 & 10 & 2.2 & 15 \\
\hline 63 & 10.94 & 26.6 & 17.3 & 28.5 & 18.8 & 74 & 72 & 170.4 & 103.6 & 50 & 12.2 & 8 \\
\hline 64 & 4.93 & 26.6 & 17.3 & 28.5 & 18.8 & 74 & 72 & 170.4 & 103.6 & 50 & 12.2 & 8 \\
\hline 65 & 30.33 & 28.5 & 18.8 & 30.1 & 21.2 & 77 & 75 & 103.6 & 148.7 & 30 & 3.9 & 30 \\
\hline 66 & 15.34 & 30.1 & 21.2 & 29.6 & 20.1 & 75 & 70 & 148.7 & 24.3 & 20 & 0.9 & 11 \\
\hline 67 & 24.88 & 29.6 & 20.1 & 32.0 & 21.4 & 70 & 66 & 24.3 & 63.0 & 20 & 2.1 & 55 \\
\hline 68 & 5.74 & 25.3 & 14.5 & 19.1 & 10.4 & 71 & 81 & 75.1 & 43.5 & 50 & 12.2 & 8 \\
\hline 69 & 29.47 & 25.3 & 14.5 & 19.1 & 10.4 & 71 & 81 & 75.1 & 43.5 & 20 & 2.1 & 19 \\
\hline 70 & 30.98 & 19.1 & 10.4 & 17.7 & 6.9 & 81 & 83 & 43.5 & 170.8 & 30 & 3.9 & 30 \\
\hline 71 & 35.24 & 19.1 & 10.4 & 17.7 & 6.9 & 81 & 83 & 43.5 & 170.8 & 20 & 0.9 & 11 \\
\hline 72 & 29.13 & 19.1 & 10.4 & 17.7 & 6.9 & 81 & 83 & 43.5 & 170.8 & 50 & 12.2 & 8 \\
\hline 73 & 3.67 & 20.5 & 11.6 & 20.0 & 12.6 & 77 & 82 & 21.0 & 234.5 & 50 & 12.2 & 8 \\
\hline 74 & 1.99 & 20.0 & 12.6 & 24.5 & 13.3 & 82 & 69 & 234.5 & 130.4 & 50 & 6.9 & 50 \\
\hline 75 & 3.09 & 20.0 & 12.6 & 24.5 & 13.3 & 82 & 69 & 234.5 & 130.4 & 20 & 2.1 & 19 \\
\hline 76 & 24.95 & 24.5 & 13.3 & 27.0 & 15.5 & 69 & 66 & 130.4 & 130.1 & 50 & 12.2 & 8 \\
\hline 77 & 23.58 & 24.5 & 13.3 & 27.0 & 15.5 & 69 & 66 & 130.4 & 130.1 & 20 & 0.9 & 11 \\
\hline 78 & 25.99 & 24.5 & 13.3 & 27.0 & 15.5 & 69 & 66 & 130.4 & 130.1 & 10 & 3.6 & 3 \\
\hline 79 & 28.66 & 24.5 & 13.3 & 27.0 & 15.5 & 69 & 66 & 130.4 & 130.1 & 30 & 3.9 & 30 \\
\hline
\end{tabular}

\section{RESULTS}

Despite extensive sampling, involving more than 136 individual samplings and 33,650 flies, only about 613 (about 2\%) D. kikkawai were captured in the two years of the study in Porto Alegre (tab. II). This species was present in each of the three urbanization zones of Porto Alegre, although it was most abundant in highly urbanized areas. Climatic conditions such as temperature, relative humidity and rainfall during the 


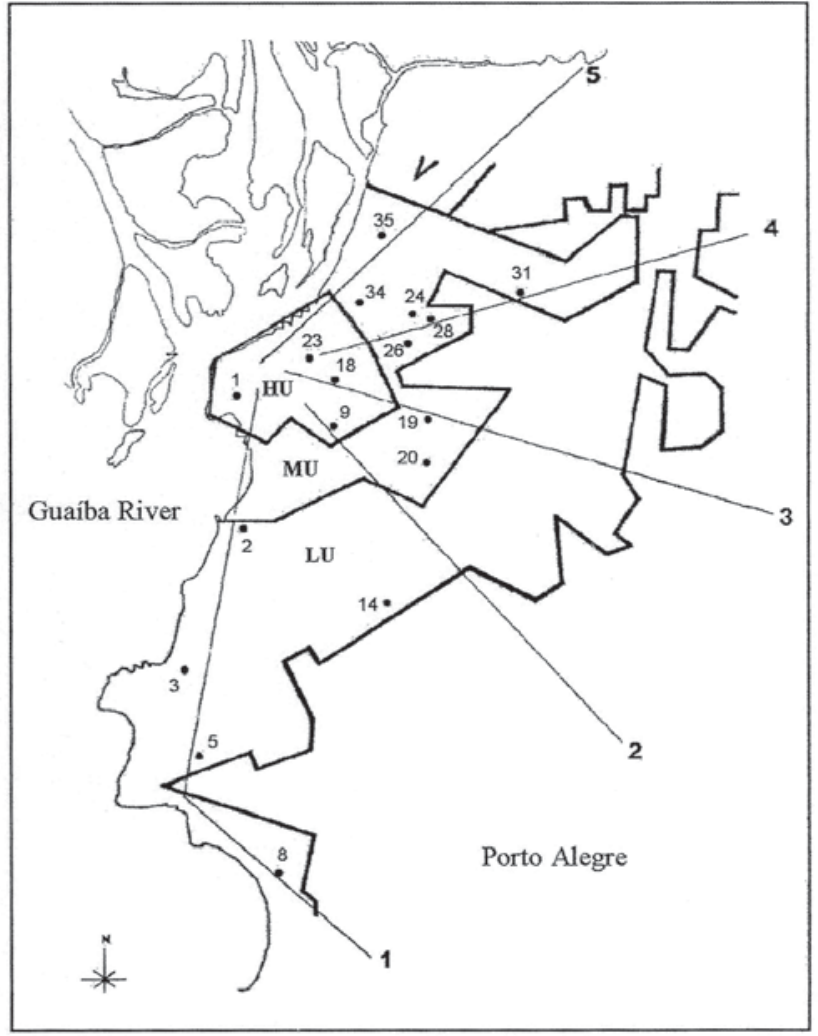

Fig. 1. Sampling sites of Porto Alegre city: 1. Fernando Machado Sq.; 2. Dona Amélia St.; 3. Veleiros do Sul Club; 5. Mario Totta St.; 8. Oswaldo Cruz St.; 9. Piratini Sq.; 14. Teresopolis St.; 18. Farroupilha Sq.; 19. Lucas de Oliveira St.; 20. Itaboraí St.; 23. Conceição St.; 24. Hilário Ribeiro St.; 26. Goethe Sq.; 28. Dona Laura St.; 31. Darcy Vignoli Sq.; 34. Visconde do Rio Branco St.; 35. Pinheiro Machado Sq. Transects (1-5) beginning in the center and radiating to the periphery of the city (HU, high urbanization; MU, medium urbanization; LU, low urbanization, according to RUszczYK (1986/1987)).

month of collection and the preceding month, as well as factors related to the urbanization of the sampling site such as percentage vegetation cover, distance from the center of the city and altitude were also considered for statistical purpose.

Multiple regression analysis was performed using the frequency of occurrence of D. kikkawai as the dependent variable versus all the other variables (urbanization and climatic data and collection date). No significant correlation results were obtained ( $\mathrm{F}=$ $1.60658 ; p>0.25<0.10$ ) for samples 45 to 79 , in spite of the tendency observed for species distribution in the urbanized zones (figs. 8-10). For these samples, however, only percentage of vegetation cover and the amount of built-up land used as co-variables were significant, suggesting a more specific role for these variables in the distribution of D. kikkawai. It seems that D. kikkawai was capable of exploiting several types of breeding sites, including native plants (Butia eriospatha (C. Mart. ex Drude) Becc., 
Table II. Characterization of Drosophila kikkawai samples in Porto Alegre city. Number and abdominal color phenotypes of flies emerged in the laboratory (P) and of the next generation (G1) (S, summer; A, autumn; W, winter; SP, spring; HU, high urbanization; MU, medium urbanization; LU, low urbanization; $\mathrm{N}$, total flies; FCC, FCc and Fcc, absolute frequency of CC, Cc and cc females, respectively; \% FCC, \% $\mathrm{FCc}$ and \% Fcc, relative frequency of $\mathrm{CC}, \mathrm{Cc}$ and $\mathrm{cc}$ females, respectively; - no flies detected).

\begin{tabular}{|c|c|c|c|c|c|c|c|c|c|c|c|c|c|c|c|c|c|c|c|}
\hline \multirow{4}{*}{$\begin{array}{l}\mathrm{S} \\
\mathrm{a} \\
\mathrm{m} \\
\mathrm{p} \\
1 \\
\mathrm{e}\end{array}$} & \multirow{4}{*}{$\begin{array}{l}\mathrm{S} \\
\mathrm{e} \\
\mathrm{a} \\
\mathrm{s} \\
\mathrm{o} \\
\mathrm{n}\end{array}$} & \multirow[t]{4}{*}{$\mathrm{T}$} & \multirow{4}{*}{$\begin{array}{l}\mathrm{U} \\
\mathrm{r} \\
\mathrm{b} \\
\mathrm{a} \\
\mathrm{n} \\
\mathrm{i} \\
\mathrm{z} \\
\mathrm{a} \\
\mathrm{t} \\
\mathrm{i} \\
\mathrm{o} \\
\mathrm{n}\end{array}$} & \multicolumn{16}{|c|}{ Generation } \\
\hline & & & & \multicolumn{8}{|c|}{$\mathrm{P}$} & \multicolumn{8}{|c|}{ G1 } \\
\hline & & & & \multirow[b]{2}{*}{$\mathrm{N}$} & \multirow[t]{2}{*}{ M } & \multicolumn{6}{|c|}{ Females } & \multirow{2}{*}{\multicolumn{2}{|c|}{ les }} & \multicolumn{6}{|c|}{ Females } \\
\hline & & & & & & FCC & $\begin{array}{c}\% \\
\text { FCC }\end{array}$ & FCc & $\begin{array}{c}\% \\
\text { FCc }\end{array}$ & Fcc & $\begin{array}{c}\% \\
\text { Fcc }\end{array}$ & & & FCC & $\begin{array}{c}\% \\
\text { FCC }\end{array}$ & FCc & $\begin{array}{c}\% \\
\text { FCc }\end{array}$ & Fcc & $\begin{array}{c}\% \\
\text { Fcc }\end{array}$ \\
\hline 01 & $\mathrm{~S}$ & 5 & MU & 10 & 4 & 1 & 17 & 2 & 33 & 3 & 50 & 289 & 143 & 29 & 20 & 72 & 49 & 45 & 31 \\
\hline 02 & $\mathrm{~S}$ & 4 & LU & 1 & - & - & - & 1 & 100 & - & - & - & - & - & - & - & - & - & - \\
\hline 03 & A & 1 & LU & 19 & 6 & - & - & 4 & 31 & 9 & 69 & 313 & 181 & 23 & 17 & 66 & 50 & 43 & 32 \\
\hline 04 & A & 2 & $\mathrm{HU}$ & 32 & 19 & - & - & 3 & 23 & 10 & 77 & 249 & 133 & 17 & 15 & 65 & 56 & 34 & 29 \\
\hline 05 & A & 1 & $\mathrm{HU}$ & 3 & 17 & - & - & 1 & f & 14 & 93 & 329 & 192 & 16 & 12 & 85 & 62 & 36 & 26 \\
\hline 06 & A & 3 & MU & 34 & 18 & - & - & 7 & 44 & 0 & 56 & 233 & 131 & 14 & 14 & 59 & 58 & 29 & 28 \\
\hline 07 & A & 1 & LU & 5 & 1 & - & - & - & - & 4 & 100 & 99 & 73 & - & - & 20 & 77 & 6 & 23 \\
\hline 08 & A & 1 & LU & 15 & 5 & - & - & 4 & 40 & 6 & 60 & 243 & 108 & 18 & 13 & 87 & 64 & 30 & 22 \\
\hline 09 & A & 4 & MU & 1 & 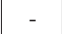 & - & - & - & - & 1 & 100 & 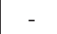 & - & - & - & - & - & - & - \\
\hline 10 & A & 3 & $\mathrm{HU}$ & 8 & 4 & - & - & 1 & 25 & 3 & 75 & 55 & 33 & 3 & 14 & 15 & 68 & 4 & 18 \\
\hline 11 & A & 1 & LU & 1 & 1 & - & - & - & - & 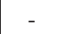 & - & 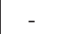 & - & - & - & - & - & - & - \\
\hline 12 & A & 1 & LU & 4 & 1 & - & - & 2 & 67 & 1 & 33 & 60 & 27 & 4 & 12 & 23 & 70 & 6 & 18 \\
\hline 13 & A & 4 & $\mathrm{HU}$ & 96 & 40 & - & - & 15 & 27 & 41 & 73 & 402 & 211 & 39 & 20 & 108 & 57 & 44 & 23 \\
\hline 14 & A & 4 & MU & 58 & 28 & - & - & 8 & 27 & 22 & 73 & 342 & 193 & 28 & 19 & 84 & 56 & 37 & 25 \\
\hline 15 & A & 1 & MU & 9 & 3 & - & - & - & 83 & 1 & 17 & 92 & 33 & 12 & 20 & 32 & 54 & 15 & 25 \\
\hline 16 & W & 4 & $\mathrm{HU}$ & 13 & 4 & - & - & 6 & 67 & 3 & 33 & 170 & 87 & 19 & 23 & 43 & 52 & 21 & 25 \\
\hline 17 & W & 4 & MU & 5 & 3 & - & - & 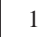 & 50 & 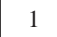 & 50 & 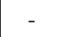 & - & - & - & - & - & - & - \\
\hline 18 & SP & 5 & MU & 2 & 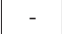 & - & - & 2 & 100 & 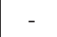 & - & 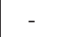 & - & - & - & - & - & - & - \\
\hline 19 & $\mathrm{~S}$ & 1 & LU & 11 & 7 & - & - & 4 & 100 & - & - & 289 & 111 & 29 & 16 & 92 & 52 & 57 & 32 \\
\hline 20 & $\mathrm{~S}$ & 1 & LU & 1 & 1 & - & - & - & - & 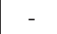 & - & - & 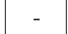 & 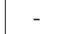 & 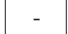 & - & - & - & - \\
\hline 21 & $\mathrm{~S}$ & 3 & M & 11 & 4 & 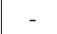 & 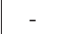 & 7 & 100 & - & - & 311 & 137 & 21 & 12 & 103 & 59 & 50 & 29 \\
\hline 22 & S & 4 & $\mathrm{HL}$ & 2 & 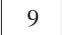 & - & - & 12 & 100 & - & - & 254 & 107 & 11 & 7 & 79 & 54 & 57 & 39 \\
\hline 23 & A & 4 & M & 2 & 9 & 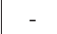 & 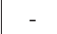 & 7 & 54 & 5 & 38 & 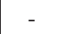 & - & - & - & - & - & - & - \\
\hline 24 & A & 1 & LL & 4 & 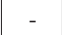 & 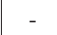 & - & 4 & 100 & - & - & - & - & - & - & - & - & - & - \\
\hline 25 & A & 4 & $\mathrm{H}$ & 16 & 6 & 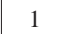 & 10 & 7 & 7 & 2 & 20 & 243 & 111 & 27 & 21 & 66 & 50 & 39 & 29 \\
\hline 26 & W & 3 & $M$ & 2 & 9 & 1 & 7 & 9 & 7 & 3 & & 32 & 159 & 36 & 21 & 88 & 51 & 49 & 28 \\
\hline 27 & W & 4 & HL & 1 & 7 & 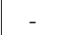 & - & 6 & 6 & 3 & & 3 & 3 & 24 & 22 & 66 & 60 & 20 & 18 \\
\hline 28 & W & 1 & $\mathrm{LU}$ & 42 & 1. & 2 & 8 & 19 & 70 & 6 & 22 & 386 & 183 & 63 & 31 & 91 & 45 & 49 & 24 \\
\hline 29 & v & 1 & LU & 1 & 1 & - & - & - & - & - & - & - & - & - & - & - & - & - & - \\
\hline 30 & $\mathrm{~S}$ & 2 & LU & 1 & - & - & - & 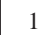 & 100 & - & 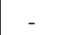 & - & - & - & - & - & - & - & - \\
\hline 31 & SP & 4 & $\mathrm{HU}$ & 1 & 1 & - & - & 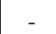 & 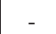 & - & - & 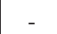 & - & - & - & - & - & - & - \\
\hline 32 & SP & 1 & LU & 20 & r & - & - & 6 & 55 & 5 & 45 & 277 & 124 & 32 & 21 & 70 & 46 & 51 & 33 \\
\hline 33 & SP & 4 & $\mathrm{HU}$ & 26 & 11 & - & - & 8 & 53 & 7 & 47 & 321 & 149 & 34 & 20 & 91 & 53 & 47 & 27 \\
\hline 34 & SP & 5 & MU & 19 & 5 & - & - & 0 & 57 & 6 & 43 & 335 & 187 & 10 & 7 & 79 & 53 & 59 & 40 \\
\hline 35 & SP & 3 & MU & 35 & 17 & - & - & 11 & 61 & 7 & 39 & 363 & 168 & 35 & 18 & 107 & 55 & 53 & 27 \\
\hline & $\Sigma$ & & & 613 & 265 & 5 & & 171 & & 172 & & 6210 & 3094 & 544 & & 1691 & & 881 & \\
\hline
\end{tabular}




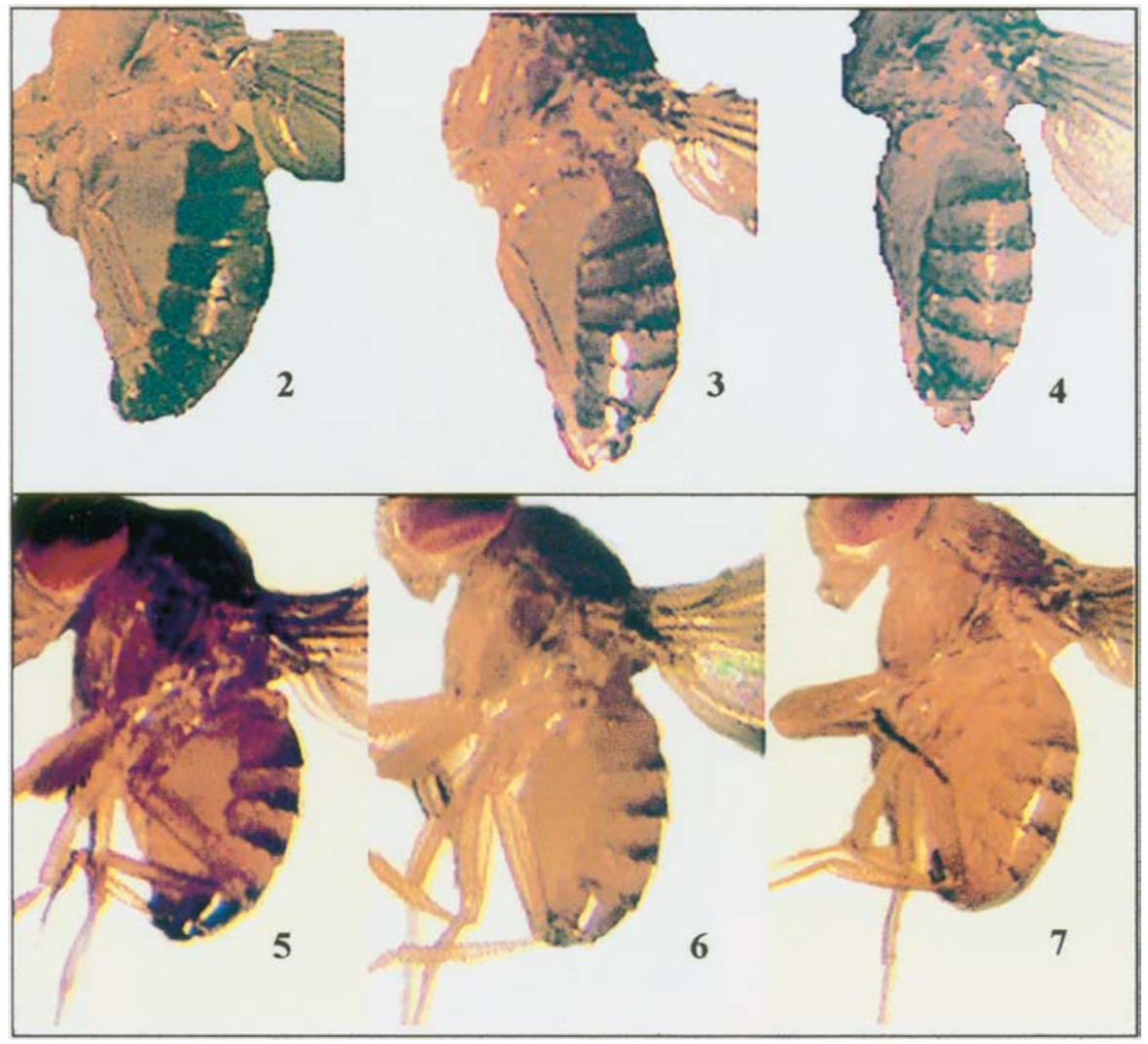

Figs. 2-7. Pigmentation polymorphism phenotypes of Drosophila kikkawai: 2, dark $\subsetneq ; 3$, medium $\subsetneq$; 4, light $\%$; 5, dark o; 6, medium o; 7, light $\sigma^{7}$.

Eugenia edulis Benth. \& Hook. f. ex Griseb., Ficus organensis Miq., Psidium guajava L. and Syagrus romanzoffiana (Cham.) Glassman), exotic plants (Averrhoa carambola L., Citrus reticulata Blanco, Citrus sinensis (L.) Osbeck, Diospyros kaki L. f., Eriobotrya japonica (Thunb.) Lindl. and Prunus persica (L.) Batsch) and garbage, as well as the banana baits where the adult flies were captured.

The frequency distribution of the three phenotypic classes of first generation offspring of flies (and their respective inferred genotypes) collected during the study (tab. II, fig. 9) showed that the effect of temperature on abdominal color polymorphism occurred in the wild D. kikkawai populations. Dark phenotypes were the most common during the coldest months (May to August) and the light phenotypes during hot months (December to March). This tendency was specially clear in samples of 1988.

When abdominal polymorphism was analyzed in relation to the urbanization levels of origin, a gradient was obtained (fig. 10) showing that darker forms (CC and $\mathrm{Cc}$ ) are associated with less urbanized areas.

For the laboratory experiments on the effects of temperature on body color, 
Table III. Phenotypic absolute number (both sexs) and gene frequency (\%) of alelles $\mathrm{C}$ and $\mathrm{c}$ in relation to abdominal tergite pigmentation polymorphism of Drosophila kikkawai maintained in chambers at $25^{\circ} \mathrm{C}$ and $17^{\circ} \mathrm{C}$, during 6 generations (G1-G6) (CC, dark; Cc, medium; cc, light; $\mathrm{N}$, total flies analyzed per generation; $\mathrm{P}$, parental generation).

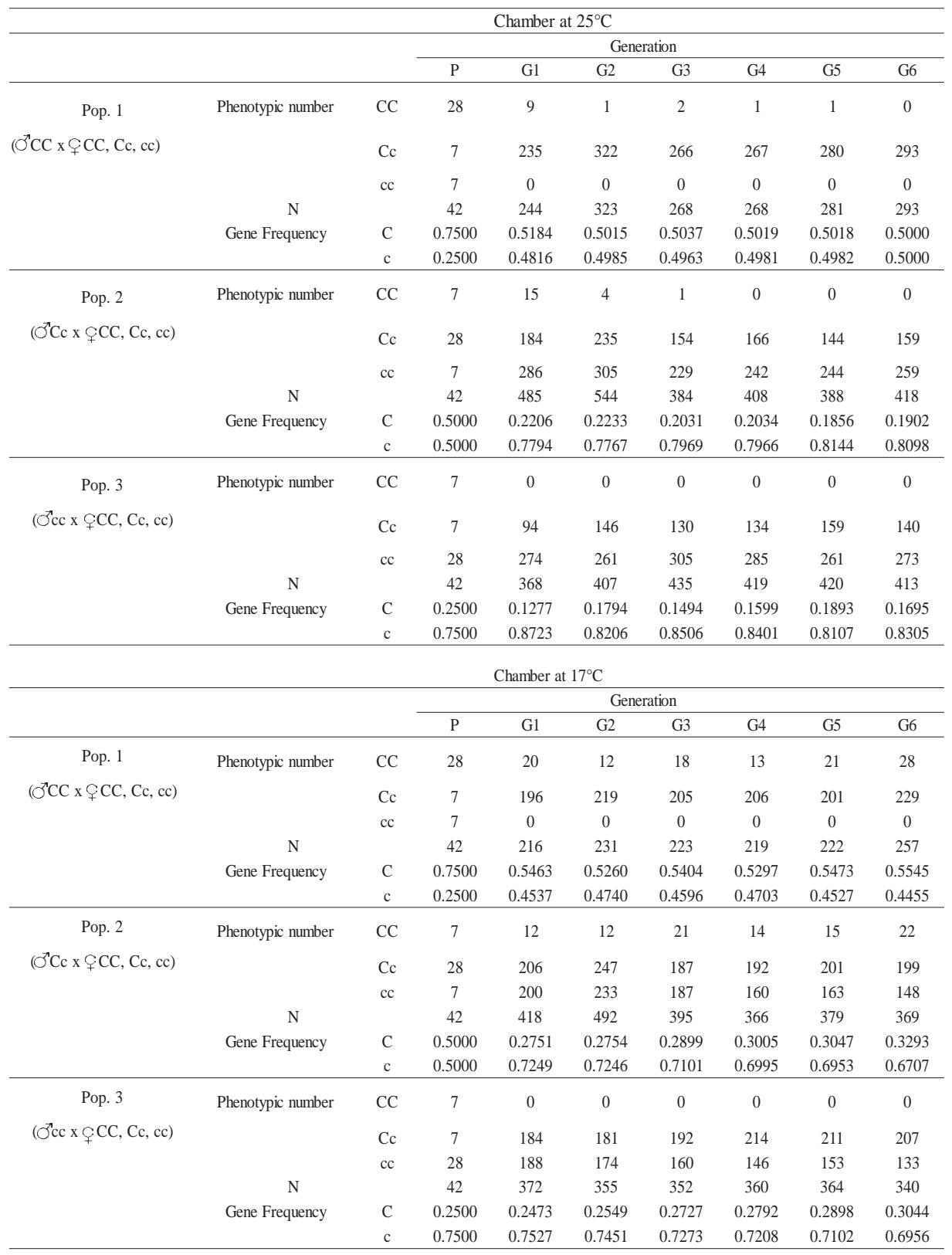




\section{$\mathrm{F}(\%)$}

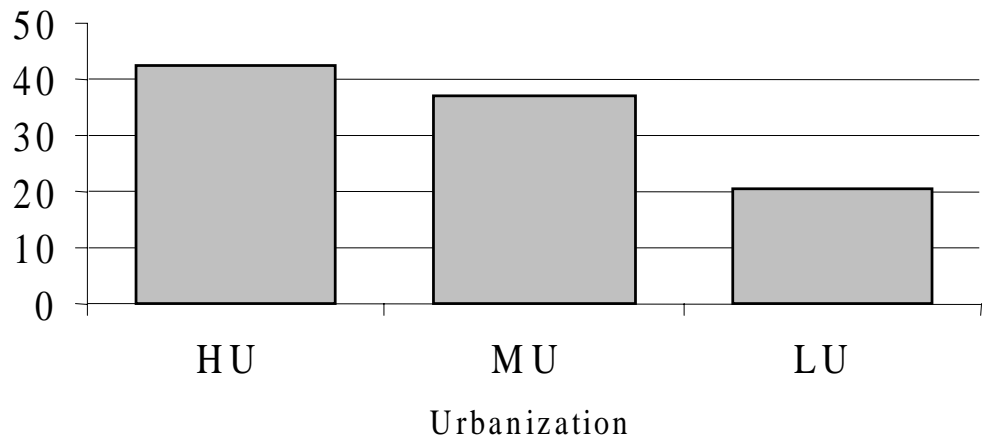

Fig. 8. Frequency of Drosophila kikkawai in the parental (P) generation for each urbanization level during the period from January 1987 to December 1988 (HU, high urbanization; MU, medium urbanization; LU, low urbanization).

$\mathrm{F}(\%)$

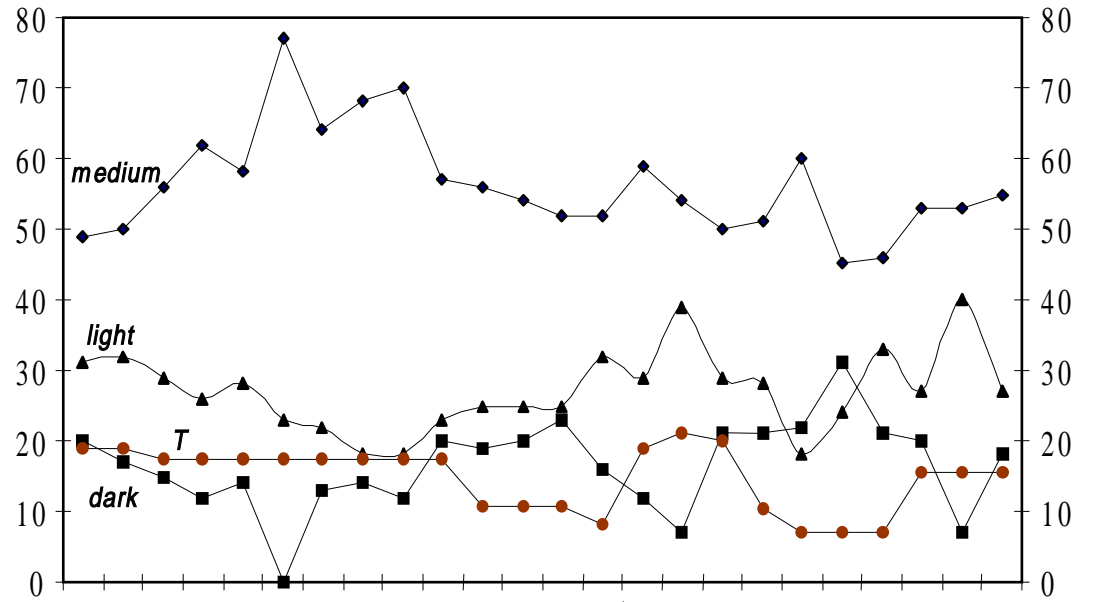

S A A A A A A A A A A A W SP 19871988

Fig. 9. Phenotype distribution ( medium; $\triangle$ light; $\square$ dark) of Drosophila kikkawai (G1 generation) according to seasons of collection during the years 1987 and 1988. Red dots represent the fluctuation of the average minimum temperatures (T) (S, summer; A, autumn; W, winter; SP, spring). 
differences were found in the frequencies of the $\mathrm{C}$ (dark) and c (light) alleles in the three laboratory populations (P1, P2 and P3) after six generations in all the crosses maintained at the two different temperatures (tab. III). In population 1 at $25^{\circ} \mathrm{C}$, the frequency of the $\mathrm{C}$ allele tended to drop from 75 to $52 \%$ in the first generation (G1) and maintained values around $50 \%$ until the sixth generation (G6). The same population at $17^{\circ} \mathrm{C}$ also presented a drop in the frequency of $\mathrm{C}$, in the first generation $(\mathrm{G} 1)$, although not so abrupt, with the $\mathrm{C}$ alelle varying around $55 \%$ until the sixth generation. Population 2 at $25^{\circ} \mathrm{C}$ showed an abrupt fall in the frequency of the $\mathrm{C}$ allele from 50 to $22 \%$ between the parent $(\mathrm{P})$ generation and the first generation, this frequency tending to be maintained at about $19 \%$ in subsequent generations. At $17^{\circ} \mathrm{C}$ the fall was less abrupt, with stabilization occurring in the sixth generation at a $\mathrm{C}$ allele frequency of about $33 \%$. With population 3 , the $\mathrm{C}$ allele frequency at $25^{\circ} \mathrm{C}$ decreased from 25 to $13 \%$ in the first generation and oscillated around 15 to $19 \%$ in the following generations, while at $17^{\circ} \mathrm{C}$ the initial $\mathrm{C}$ allele frequency tended to increase until it attained $30 \%$ in the sixth generation.

The tendencies observed in these experiments suggest some advantages for the $\mathrm{C}$ allele at lower temperatures. These findings are similar to those obtained for natural populations, where darker flies are more frequent in winter (fig. 9) and in less urbanized zones (fig. 10) where temperatures tend to be lower than in more urbanized zones.

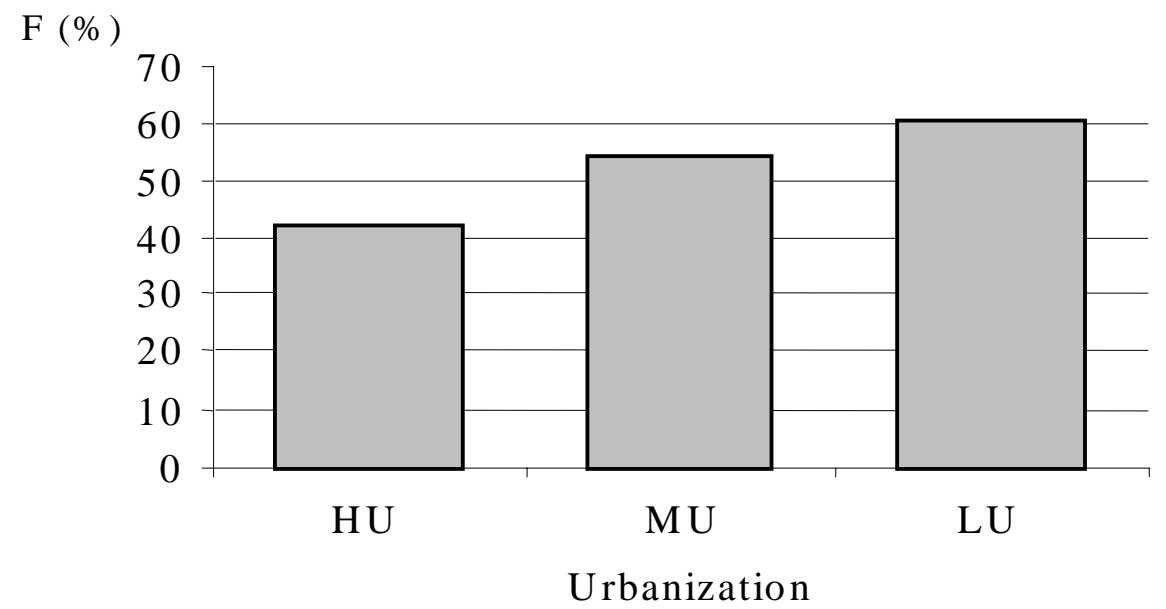

Fig. 10. Percent distribution (F \%) of darker females (CC and $\mathrm{Cc}$ ) in relation to all Drosophila kikkawai collected at each urbanization level in Porto Alegre city, in the parental (P) generation (HU, high urbanization; MU, medium urbanization; LU, low urbanization).

\section{DISCUSSION}

In spite of the small number of Drosophila kikkawai specimens in the Porto Alegre samples, its presence was constant during the collection period, unlike other wild species such as Drosophila willistoni Sturtevant, 1916, Drosophila nebulosa Sturtevant, 1916, and Drosophila paulistorum Dobzhansky \& Pavan, 1943 whose populations have shown clear peaks during several years of observation (VALENTE et al., 1989). 
If neither climatic factors nor variations in the urban environment contribute exclusively to the maintenance of $D$. kikkawai in cities, the availability of trophic resources may be an important factor in the regulation of D. kikkawai populations. Although this fruit fly seems to prefer exotic fruits (six out of eleven plants sampled) it is also successful in exploiting the native flora ( 5 plants). We found a weak association between the numbers of D. kikkawai and the more urbanized areas of Porto Alegre in which there is little vegetation cover and therefore a lower availability of naturally occurring fruits. This suggests that this species can successfully exploit substrates provided by man, such as garbage. Taken together, these findings suggest the recent introduction of $D$. kikkawai to this location and support its classification as a cosmopolitan species (PINHEIRO \& VALENTE, 1993); LEMEUNIER et al. (1986) having attributed the dispersion of $D$. kikkawai exclusively to human activity.

The present results on pigmentation polymorphism of the posterior abdominal segments of D. kikkawai support those of Freire-Maia (1963, 1964a, b) and FreireMAIA \& FREIRE-MAIA (1964) who showed that heterozygotes for this polymorphism were the best adapted genotype, characterizing a type of balanced polymorphism. In high temperature locations, Freire-Maia (1949, 1953), Freire-Maia et al. (1954) and FreireMAIA \& Freire-MAiA (1964) also observed that light phenotypes were more common, whereas the darker forms appeared to be favored at places having lower temperatures. Their studies, however, were based on a survey of geographical variation at various sites along the Brazilian coast during the same season. Although the study reported in the present paper relies on data obtained by observation of seasonal variation over a two-year period, they reinforce the results obtained by FREIRE-MAIA (1949, 1953).

Other evidence of temperature effects on this polymorphism in natural populations of D. kikkawai comes from the analysis of the association between the frequencies of darker forms and low urbanization levels of the sampling sites. If we consider that zones of higher urbanization are considerably warmer than peripheral zones (DUCKWORTH \& SANDBERG, 1954), these findings support the idea that darker phenotypes are well suceeded in low temperatures. According to BRYSON \& Ross (1972) the well-documented climatic differences between cities and their surroundings are due to a combination of the extensive road cover, less air circulation caused by buildings and air pollution, all of which contribute to turning the city into a 'thermal island'. The gradient observed in the frequencies of the color patterns of D. kikkawai from the center to the periphery of Porto Alegre, may reflect a temperature gradient imposed by the radial urbanization characteristic of Porto Alegre and suggests that the difference of a few degrees of temperature caused by different levels of urbanization is enough to favor different phenotypes in each urban zone.

The temperatures used in laboratory experiments $\left(17^{\circ}\right.$ and $\left.25^{\circ} \mathrm{C}\right)$ did not appear to be sufficient to promote strong differences in gene frequencies in the D. kikkawai populations analyzed. GIBERT et al. (1999) found color differences in Indian populations of D. kikkawai subjected to seven other experimental temperatures from $12^{\circ}$ to $30^{\circ} \mathrm{C}$. In fact, in Porto Alegre environmental temperatures are considerably lower than $17^{\circ} \mathrm{C}$ in winter (average minimum temperature of about $8^{\circ} \mathrm{C}$ ) and higher than $25^{\circ} \mathrm{C}$ in the summer (average maximum temperature of about $30^{\circ} \mathrm{C}$ ). Despite these differences between the experimental conditions and those found in the Porto Alegre environment and the limited number of generations analyzed, we still observed opposite tendencies in the frequencies 
of the $\mathrm{C}$ and $\mathrm{c}$ alleles in laboratory populations kept at $17^{\circ}$ and $25^{\circ} \mathrm{C}$. These finding suggests once again that the allele determining dark body color is favored at lower temperatures.

Color polymorphism in abdominal segments is not an uncommon phenomenon in Drosophila (review in Payant, 1986), and has been studied by Da Cunha (1949), HeEd \& Blake (1963), Napp Martinez \& Cordeiro (1970) and Machado et al. (2001) in South American Drosophila polymorpha Dobzhansky \& Pavan, 1943. DA CunHA (1949) found that genes with a different mode of action are responsible for the control of the expression of abdominal pigmentation in populations of the northern part of South America compared with those from the rest of Brazil. These latitudinal differences may reflect, once again, a selective response to different temperatures. Perhaps such latitudinal differences can also explain the different pattern of phenotype variation found by GIBERT et al. (1999) in Indian populations of D. kikkawai compared with those found in Brazilian populations of this fly.

The physiological basis for the advantage of darker forms of D. kikkawai in winter months and in colder environments is still not completely clear. The association between darker bodies and lower temperatures (and vice-versa) can be explained by the thermal budget hypothesis, which states that dark bodies absorb solar radiation better than those of light ones. Consequently, darker forms are favored in their sexual performances, activities of locomotion and in flight when they are subject to low temperatures.

Acknowledgments. To Nena Basílio Morales and Vera M. Rodrigues for technical support and the Brazilian agencies CNPq, FAPERGS, PROPESQ-UFRGS and FINEP for fellowships and grants.

\section{REFERENCES}

Ashburner, M. 1989. The melanogaster species group. In: Ashburner, M. ed. Drosophila, a Laboratory Handbook. New York, Cold Spring Harbor Laboratory. p.1161-1166.

Baimai, V.; Traipakvasin, A. \& Kitagawa, O. 1986. Additional data on metaphase karyotype variation and geographic distribution of the Drosophila kikkawai complex. Jpn. J. Genet., Tokyo, 61(3):207-216.

Bryson, R. A. \& Ross, J. E. 1972. The climate of the city. In: Detwyler, T. \& Marcus, M.G. eds. Urbanization and Environment. The physical geography of the city. Massachussets, Duxbury. p.51-68.

Da Cunha, A. B. 1949. Genetic analysis of the polymorphism of color pattern in Drosophila polymorpha. Evolution, Lancaster, 3:239-251.

Duckworth, E. S. \& Sandberg, J. S. 1954. The effect of cities upon horizontal and vertical temperature gradients. Bull. Am. met. Soc., Boston, 35:198-207.

Freire-Maia, N. 1949. Balanced polymorphism in Drosophila montium. Evolution, Lancaster, 3:98.

_- 1953. Frequencies of the two color forms of the Brazilian Drosophila montium in natural populations. Drosoph. Inf. Serv., Norman, 27:90, 91.

_.. 1963. Carga genética, o preço da evolução. In: Pavan, C. \& Da Cunha, A. B. eds. Genética. Aspectos modernos da Genética Pura e Aplicada. São Paulo, Cia Editora Nacional. p. $290-317$.

1964a. Segregational load in Drosophila kikkawai. I. Crossing experiments. Genetics, Austin, 50:211-219.

1964b. Segregational load in Drosophila kikkawai. II. Experimental populations. Genetics, Austin, 50:221-229. 
Freire-Maia, N. \& Freire-Maia, A. 1964. Segregational load in Drosophila kikkawai. III. Natural populations. Genetics, Austin, 50:789-802.

Freire-Maia, N.; Freire-Maia, A. et al. 1954. The Hardy-Weinberg law in Brazilian natural populations of Drosophila kikkawai. Drosoph. Inf. Serv., Norman, 28:119, 120.

Gibert, P.; Moreteau, B. et al. 1996. Growth temperature and adult pigmentation in two Drosophila sibling species: an adaptive convergence of reaction norms in sympatric populations. Evolution, Lancaster, 50:2346-2353.

Gibert, P.; Moreteau, B. et al. 1998. Ligth body pigmentation in Indian Drosophila melanogaster: a likely adaptation to a hot and arid climate. J. Genet., Cambridge, 77:13-20.

Gibert, P.; Moreteau, B. et al. 1999. Phenotypic plasticity of abdominal pigmentation in Drosophila kikkawai: multiple interactions between a major gene, sex, abdomen segment and growth temperature. Genetica, Dordrecht, 105:165-176.

Heed, W. B. \& Blake, P. R. 1963. A new color allele at the "e" locus of Drosophila polymorpha from northern south America. Genetics, Austin, 48:217-234.

LEE, T. J. 1963. Genetic analysis of the polymorphism of color pattern in Drosophila auraria. Drosoph. Inf. Serv., Norman, 37:97, 98.

Lemeunier, F.; David, J. R. et al. 1986. The melanogaster species group. In: AshbuRner, M.; Carson, H. L. \& Thompson, J. N., JR. eds. The genetics and biology of Drosophila. London, Academic. v.3e, p.147-256.

Machado, M. X.; Toni, D. C. DE \& Hofmann, P. R. P. 2001. Abdominal pigmentation polymorphism of Drosophila polymorpha (Dobzhansky and Pavan, 1943) collected on Ilha de Santa Catarina and neighboring islands. Biotemas, Florianópolis, 14(1):87-107.

Marques, E. K.; NAPP, M. et al. 1966. A corn meal, soybean flour, wheat germ medium for Drosophila. Drosoph. Inf. Serv., Norman, 41:187.

Napp Martinez, M. \& Cordeiro, A. R. 1970. Modifiers of color pattern genes in Drosophila polymorpha. Genetics, Austin, 64:573-587.

Payant, V. 1986. Le polymorphisme de la coloration abdominale dans le genre Drosophila. Annee biol., Paris, 25(2):167-184.

Pinheiro, B. E. C. \& Valente, V. L. S. 1993. Drosophila kikkawai, a subcosmopolitan or a cosmopolitan species? Drosoph. Inf. Ser., Norman, 72:146-149.

RuszczyK, A. 1986/1987. Distribution and abundance of butterflies in the urbanization zones of Porto Alegre, Brazil. J. Res. Lepidop., Beverly Hills, 25(3):157-178.

Tidon, R. \& Sene, F. M. 1988. A trap that retains and keeps Drosophila alive. Drosoph. Inf. Serv., Norman, 67:89.

TsacAs, L. \& DAVID, J. R. 1978. Systematics and biogeography of the Drosophila kikkawai complex, with descriptions of new species (Diptera, Drosophilidae). Annls Soc. ent. Fr., Paris, 13:675-693.

Valente, V. L. S.; Ruszczyk, A. et al. 1989. Genetic and ecological studies on urban and marginal populations of Drosophila in the south of Brazil. Evol. Biol., Bogotá, 3:19-35.

Recebido em 31.10.2002; aceito em 04.07.2003. 